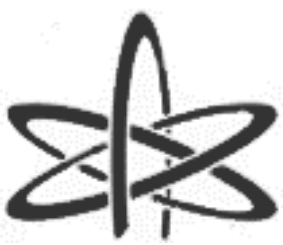

BJRS

\author{
BRAZILIAN JOURNAL \\ $\mathrm{OF}$ \\ RADIATION SCIENCES \\ 07-2A (2019) 01-12
}

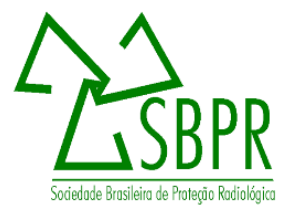

\title{
Assessment of Sr-90 in water samples: precision and accuracy
}

\author{
M. B. Nisti ${ }^{\text {a }}$ C. H. R. Saueia ${ }^{\text {aa }}$ B. Castilho ${ }^{\text {a. }}$ B. P. Mazzilli ${ }^{\text {a }}$ \\ ${ }^{a}$ Instituto de Pesquisas Energéticas e Nucleares (IPEN / CNEN - SP) \\ Postal code 05508-000, São Paulo, SP, Brazil \\ mbnisti@ipen.br
}

\begin{abstract}
The aims of this study are to evaluate the precision and accuracy of three methodologies for the determination of ${ }^{90} \mathrm{Sr}$ in water samples: Cerenkov, LSC direct method and with radiochemical separation. The performance of the methodologies was evaluated by using two scintillation counters (Quantulus and Hidex). The parameters Minimum Detectable Activity (MDA) and Figure Of Merit (FOM) were determined for each method, the precision and accuracy were checked using ${ }^{90} \mathrm{Sr}$ standard solutions.
\end{abstract}

Keywords: LSC; Cerenkov; ${ }^{90} \mathrm{Sr}$; precision; accuracy. 


\section{INTRODUCTION}

The study of artificial radionuclides dispersion into the environment is very important to control the nuclear waste discharges, nuclear accidents and nuclear weapons testing. The ${ }^{90} \mathrm{Sr}$ is produced by nuclear fission with a physical half-life of 28.79 years with decay energy of $0.546 \mathrm{MeV}$. Due to its long physical and biological half -lives, ${ }^{90} \mathrm{Sr}$ is normally found in the environment in equilibrium with its ${ }^{90} \mathrm{Y}$ daughter, with a physical half-life of 64.05 hours with maximum energy of $2.280 \mathrm{MeV}$ [1]. ${ }^{90} \mathrm{Sr}$ is very important to study it can deposit in the bone by its chemical similarity with calcium. The measurement of ${ }^{90} \mathrm{Sr}$ by Liquid Scintillation Counting (LSC) presents several advantages: high efficiency of detection, easy sample preparation techniques, automation and simultaneous analysis of different radionuclides [1,2].

LSC is a technique in which the sample is mixed to the liquid scintillation, forming a scintillation solution, capable of converting the kinetic energy of nuclear emissions into light photons. The interaction of the emissions occurs in the liquid solution, producing excitement with emission of photons of ultraviolet radiation [2].

When the velocity of a charged particle in a transparent medium exceeds the speed of light, the resulting radiation produced is called Cerenkov Effect. When produced at significant levels, Cerenkov radiation can be employed for the measurement of radioactivity. Some radionuclides may be counted directly in water, the advantages are: no need of scintillation cocktail, sample suitable for any other chemical tests; simplicity of sample preparation; low cost; easy discard and efficiency unaffected by chemical quenching. The disadvantages are: the method is applicable only to beta emitters; energies above the threshold and the counting efficiency is lower when compared with LSC. The threshold energy for production of Cerenkov photons by electrons or beta particles is 262 $\mathrm{keV}[2]$.

When the sample to be analyzed consists of two radionuclides, the Cerenkov method can be used combined with LSC method. Water is the usual sample for determination of ${ }^{90} \mathrm{Sr}[2]$.

The aims of this study is to evaluate the precision and accuracy of three methodologies for the determination of ${ }^{90} \mathrm{Sr}$ in water samples: Cerenkov, liquid scintillation counting (LSC) direct method and with radiochemical separation. The parameters Minimum Detectable Activity (MDA) and 
Figure of Merit (FOM) were determined for each method; the precision and accuracy were checked using ${ }^{90} \mathrm{Sr}$ standard solutions.

\section{MATERIALS AND METHODS}

The performance of the methodologies was evaluated by using two scintillation counters (Quantulus and Hidex). The equipment 1220 Quantulus ${ }^{\mathrm{TM}}$ Ultra Low Level Liquid Scintillation Spectrometer is composed by two photomultipliers and has the advantage of having an extremely low background for performing radioactivity measurements, while the low-level Hidex 300-SL, is composed by three photomultipliers $[3,4]$.

The counting efficiency is the ratio of counts per number of decay events, which occur during the measuring time [5].

The Quantulus counting efficiency was determined using ${ }^{90} \mathrm{Sr}$ standard solution, according to equation (1) [5].

$$
E f=\left(\frac{\text { counts }-B g}{\text { Activity }}\right)
$$

Where Ef is the counting efficiency $\left(\mathrm{cps} \mathrm{dps}^{-1}\right)$, Activity is the standard solution activity (Bq), counts is the source counts (cps), and $\mathrm{Bg}$ is the background radiation (cps).

For Hidex equipment, the efficiency for ${ }^{90} \mathrm{Sr}$ was determined using Triple to Double Coincidence Ratio (TDCR), which is a primary measurement method based on calculation of the efficiency from the measured ratio of double and triple coincidence counting rates. TDCR does not require standard solution, therefore it is an absolute measurement [6].

The counting efficiency of Hidex was obtained by using the equation (2) [7].

$$
T D C R=\frac{\int_{0}^{E_{\max }} S(E)\left(1-e^{-n}\right)^{3} d E}{\int_{0}^{E_{\max }} S(E)\left(\left(3\left(1-e^{-n}\right)^{2}-2\left(1-e^{n}\right)^{3}\right)\right) d E}
$$


Where $\mathrm{S}(E)\left(1-e^{-n}\right)^{3}$ is the triple coincidences, and $\mathrm{S}(E)\left(\left(3\left(\left(1-e^{-n}\right)^{2}-2\left(1-e^{-n}\right)^{3} e^{-n}\right.\right.\right.$ is the all coincidences

\subsection{Procedures for preparing the ${ }^{90} \mathrm{Sr}$ solution}

\subsubsection{Cerenkov method}

The initial step of pre-concentration of the ${ }^{90} \mathrm{Sr}$ solution consisted of heating on a hot plate, at a temperature of $80{ }^{\circ} \mathrm{C}$, until reduction of the volume by a factor of 32. An aliquot of $20 \mathrm{~mL}$ of each sample was measured in the appropriate vial. The channel range used was from 5 to 350, for both equipment. The background was determined with ultrapure water following the same procedure. The counting time was 14,400 seconds and the sample was analyzed in triplicate for both equipment.

\subsection{2. (LSC) direct method}

The initial step of pre-concentration of the ${ }^{90} \mathrm{Sr}$ solution consisted of heating on a hot plate, at a temperature of $80{ }^{\circ} \mathrm{C}$, until reduction of the volume by a factor of 80 . An aliquot of $5 \mathrm{~mL}$ of the final solution was mixed with $15 \mathrm{~mL}$ Ultima Gold AB scintillation solution in the appropriate vial for counting. The channel range used was from 5 to 1000. The background was determined with ultrapure water following the same procedure. The counting time was 14,400 seconds and the sample was analyzed in triplicate for both equipment.

\subsection{3. (LSC) method with radiochemical separation}

A volume of $50 \mathrm{~mL}$ of the ${ }^{90} \mathrm{Sr}$ solution was concentrated by a factor of 8 . The solution was mixed with $1000 \mu \mathrm{L}$ of $\mathrm{Sr}$ carrier. The sample was evaporated and dissolved with $30 \mathrm{~mL}$ of $8 \mathrm{M} \mathrm{HNO}$ under shaking and heating. The solution was loaded onto the $\mathrm{Sr}$ resin column preconditioned in advance with $8 \mathrm{M} \mathrm{HNO}_{3}{ }^{90} \mathrm{Sr}$ was retained in the resin column and ${ }^{90} \mathrm{Y}$ was eluted, at a flow rate 12 drops $\mathrm{min}^{-1} .{ }^{90} \mathrm{Sr}$ was eluted in the resin column with $30 \mathrm{~mL}$ of $0.5 \mathrm{M} \mathrm{HNO}_{3} .0 .3 \mathrm{~g}$ of oxalic acid and drops of $\mathrm{NH}_{3}$ were added under gently shaking, strontium was precipitated. The final 
precipitate was weighed to calculate the chemical recovery using the gravimetric method. The precipitate was quantitatively transferred into an appropriate vial together with the filter paper, and $1 \mathrm{~mL}$ of $1 \mathrm{M} \mathrm{HNO}_{3}$ was added into the vial to dissolve the precipitate. Finally, $15 \mathrm{ml}$ of Ultima Gold AB scintillation solution was added to the vial. The final solution was counted. The chemical yield ranged from $64 \%$ to $87 \%$. The channel range used was from 300 to 700 .

When the water sample presents other beta emitting radionuclides, the counts is increased, affecting the accuracy [8], in this case, it is required to separate ${ }^{90} \mathrm{Sr}$ from the interfering elements by using the radiochemical separation method. The background was determined with ultrapure water following the same procedure. The counting time was 14,400 seconds and the sample was analyzed in triplicate for both equipment.

\section{RESULTS AND DISCUSSION}

\subsection{Efficiency}

The efficiency for ${ }^{90} \mathrm{Sr}$ was measured for the Quantulus using ${ }^{90} \mathrm{Sr}$ standard solution, with activity of 10.3 Bq, and for the Hidex, the efficiency was determined by using the TDCR method.

The results obtained for the efficiency for both equipment and methods are presented in Table 1.

Table 1: Efficiency for Quantulus and Hidex (cps.dps $\left.{ }^{-1}\right)$

\begin{tabular}{|c|c|c|}
\hline Method & $\begin{array}{l}\text { Quantulus } \\
\left(\text { cps.dps }^{-1}\right)\end{array}$ & $\begin{array}{c}\text { Hidex } \\
\left(\text { cps.dps }^{-1}\right) \\
\end{array}$ \\
\hline Cerenkov $^{\mathrm{a}}$ & $0.642 \pm 0.002$ & $0.606 \pm 0.002$ \\
\hline (LSC) direct method ${ }^{\mathrm{a}}$ & $1.830 \pm 0.039$ & $1.707 \pm 0.038$ \\
\hline (LSC) radiochemical separation & $0.858 \pm 0.030$ & $0.975 \pm 0.044$ \\
\hline
\end{tabular}

The efficiencies determined are in good agreement with the literature values [1,2].

For the sake of comparison, the efficiency obtained for the Hidex by the TDCR method was compared with the method using ${ }^{90} \mathrm{Sr}$ standard solution. The counting efficiency results are presented in Table 2. 
Table 2: Efficiency using the ${ }^{90} \mathrm{Sr}$ standard solution and TDCR method (cps.dps ${ }^{-1}$ )

\begin{tabular}{ccc}
\hline Sample & $\begin{array}{c}{ }^{\mathbf{9 0}} \text { Sr standard solution } \\
\left(\mathbf{c p s . d p s}^{-1}\right)\end{array}$ & $\begin{array}{c}\text { TDCR method } \\
\left(\text { cps.dps }^{-1}\right)\end{array}$ \\
\hline $\mathbf{1}$ & $0.607 \pm 0.004$ & $0.605 \pm 0.006$ \\
\hline $\mathbf{2}$ & $0.600 \pm 0.005$ & $0.606 \pm 0.004$ \\
\hline $\mathbf{3}$ & $0.599 \pm 0.005$ & $0.606 \pm 0.003$ \\
\hline mean & $0.602 \pm 0.004$ & $0.606 \pm 0.001$ \\
\hline
\end{tabular}

The efficiencies obtained by both methods were similar.

\subsection{MDA and FOM}

An approach useful for the comparison of sensibility for different methodologies is the Figure of Merit (FOM) [1].

The FOM was obtained by the equation (3) [1].

$$
F O M=\left(\frac{(E f \%)^{2}}{B g}\right)
$$

Where FOM is the figure of merit, Ef\% is the counting efficiency (\%), and $\mathrm{Bg}$ is the background count rate $(\mathrm{cpm})$.

Another important parameter in the comparison of different methodologies is the minimum detectable activity (MDA). The determination of the minimum detectable activity (MDA) followed the model proposed by Currie [9]. The MDA was determined for a counting time of 14,400 s. The results obtained for MDA and FOM for the Quantulus and Hidex equipment are presented in table 3 and 4, respectively.

Table 3: MDA and FOM for Quantulus

\begin{tabular}{ccc}
\hline Method & MDA $\left(\mathbf{B q ~ L}^{-1}\right)$ & FOM \\
\hline Cerenkov & 0.02 & 2369 \\
\hline (LSC) direct method & 0.02 & 4998 \\
\hline (LSC) radiochemical separation & 0.04 & 1963 \\
\hline
\end{tabular}


Table 4: MDA and FOM for Hidex

\begin{tabular}{ccc}
\hline Method & MDA $\left(\mathbf{B q ~ L}^{-\mathbf{1}}\right)$ & FOM \\
\hline Cerenkov & 0.06 & 147 \\
\hline (LSC) direct method & 0.07 & 317 \\
\hline (LSC) radiochemical separation & 0.09 & 302 \\
\hline
\end{tabular}

\subsection{Precision and Accuracy}

The precision and accuracy of the methodologies were checked by measuring ${ }^{90} \mathrm{Sr}$ standard solutions in trriplicate. The precision was evaluated by the equation (4).

$$
R S D=\left(\frac{S D}{A V}\right) \cdot 100
$$

Where RSD is the relative standard deviation, SD is the standard deviation, and AV is the average value.

The accuracy was evaluated by the equation (5).

$$
R E=\left(\frac{|A V-R V|}{R V}\right) \cdot 100
$$

Where $\mathrm{RE}$ is the relative error, $\mathrm{RV}$ is the reference value, and $\mathrm{AV}$ is the average value.

The results obtained for the precision and accuracy for Hidex are presented in Table 5. 
Table 5: Precision and accuracy for Hidex

\begin{tabular}{lcccccc}
\hline & \multicolumn{2}{c}{ Cerenkov } & \multicolumn{2}{c}{$\begin{array}{c}\text { (LSC) direct } \\
\text { method }\end{array}$} & \multicolumn{2}{c}{$\begin{array}{c}\text { radiochemical } \\
\text { separation }\end{array}$} \\
\hline & $\begin{array}{c}\text { Precision } \\
(\boldsymbol{\%})\end{array}$ & $\begin{array}{c}\text { Accuracy } \\
(\boldsymbol{\%})\end{array}$ & $\begin{array}{c}\text { Precision } \\
(\boldsymbol{\%})\end{array}$ & $\begin{array}{c}\text { Accuracy } \\
(\boldsymbol{\%})\end{array}$ & $\begin{array}{c}\text { Precision } \\
(\boldsymbol{\%})\end{array}$ & $\begin{array}{c}\text { Accuracy } \\
(\boldsymbol{\%})\end{array}$ \\
\hline A & 2.9 & 9.7 & 3.6 & 3.5 & 2.0 & 14.0 \\
\hline B & 2.9 & 6.9 & 16.3 & 9.3 & 8.7 & 1.6 \\
\hline C & 1.0 & 8.3 & 2.6 & 6.2 & 6.8 & 8.6 \\
\hline
\end{tabular}

The results obtained for the precision and accuracy for Quantulus are presented in Table 6.

Table 6: Precision and accuracy for Quantulus

\begin{tabular}{|c|c|c|c|c|c|c|}
\hline & \multicolumn{2}{|c|}{ Cerenkov } & \multicolumn{2}{|c|}{$\begin{array}{l}\text { (LSC) direct } \\
\text { method }\end{array}$} & \multicolumn{2}{|c|}{$\begin{array}{c}(\mathrm{LSC}) \\
\text { radiochemical } \\
\text { separation }\end{array}$} \\
\hline & $\begin{array}{c}\text { Precision } \\
(\%)\end{array}$ & $\begin{array}{c}\text { Accuracy } \\
(\%)\end{array}$ & $\begin{array}{c}\text { Precision } \\
(\%)\end{array}$ & $\begin{array}{c}\text { Accuracy } \\
(\%)\end{array}$ & $\begin{array}{c}\text { Precision } \\
(\%)\end{array}$ & $\begin{array}{c}\text { Accuracy } \\
(\%)\end{array}$ \\
\hline $\mathbf{A}$ & 1.6 & 1.6 & 1.0 & 15.8 & 15.8 & 11.4 \\
\hline B & 2.2 & 3.1 & 2.8 & 10.1 & 16.3 & 9.3 \\
\hline $\mathbf{C}$ & 1.0 & 9.4 & 1.2 & 3.7 & 0.6 & 0.3 \\
\hline
\end{tabular}

The accuracy and precision was acceptable for all the methodologies studied.

\subsection{Performance}


The performance of the three methodologies was evaluated by participating in a proficiency test organized by Instituto de Radioproteção e Dosimetria

The normalized standard deviation, D, was evaluated by the equation (6) [10].

$$
D=\left(\frac{(X-U)}{\frac{S u}{\sqrt{n}}}\right)
$$

Where $\mathrm{D}$ is the normalized standard deviation, $\mathrm{X}$ is the mean value, $\mathrm{U}$ is the reference value, $\mathrm{Su}$ is the standard deviation of the reference value, and $\mathrm{n}$ is the number of independent determinations. When $D$ (normalized standard deviation) results are within the interval $-2 \leq D \leq+2$, the performance of the laboratory is considered good, results in the intervals $+2<D<+3$ and $-3<D<$ -2 are considered acceptable, data with $D \leq-3$ or $D \geq+3$ indicate that the measurement system is out of control [10]. The results obtained are presented in Table 7.

Table 7: Performance of the methods (normalized standard deviation (D)): Cerenkov, (LSC) direct method and (LSC) radiochemical separation

\begin{tabular}{ccccccc}
\hline & \multicolumn{3}{c}{$(D)$ - Quantulus } & \multicolumn{3}{c}{$(D)$ - Hidex } \\
\hline Methodology & PT(A) & PT(B) & PT(C) & PT(A) & PT(B) & PT(C) \\
\hline Cerenkov & 0.19 & 0.35 & 1.09 & 1.12 & 0.78 & 0.96 \\
\hline (LSC) direct method & -1.84 & 1.19 & 0.43 & -0.41 & 0.18 & -0.72 \\
\hline (LSC) radiochemical separation & -1.32 & 1.09 & 0.04 & -1.63 & -0.18 & 1.01 \\
\hline
\end{tabular}

The values obtained for the normalized standard deviation (D) were always below 2, indicating good results for both equipment and methodology.

The second criteria used in the performance evaluation is the normalized deviation (E), when $|\mathrm{E}|<$ 1 the result is consistent [11]. The normalized deviation, E, was evaluated by the equation (7). 


$$
E=\left(\frac{(X-U)}{k \sqrt{S x^{2}+S u^{2}}}\right)
$$

Where $\mathrm{E}$ is the normalized deviation, $\mathrm{X}$ is the laboratory mean value, $\mathrm{Sx}$ is the standard deviation of the laboratory, $\mathrm{U}$ is the reference value, $\mathrm{Su}$ is the standard deviation of the reference value, and $\mathrm{k}$ is the coverage factor $(k=2)$.

The results obtained for the normalized deviation (E) are presented in Table 8.

Table 8: Performance of the methods (normalized deviation (E)): Cerenkov, (LSC) direct method and (LSC) radiochemical separation

\begin{tabular}{ccccccc}
\hline & \multicolumn{3}{c}{$(\boldsymbol{E})$ - Quantulus } & \multicolumn{3}{c}{$(\boldsymbol{E})$ - Hidex } \\
\hline Methodology & PT(A) & PT(B) & PT(C) & PT(A) & PT(B) & PT(C) \\
\hline Cerenkov & 0.05 & 0.10 & 0.31 & 0.32 & 0.22 & 0.28 \\
\hline (LSC) direct method & -0.53 & 0.33 & 0.12 & -0.11 & 0.05 & -0.21 \\
\hline (LSC) radiochemical separation & -0.28 & 0.20 & 0.01 & -0.47 & -0.05 & 0.26 \\
\hline
\end{tabular}

The values obtained for the normalized deviation (E) were always below 1, indicating results consistent for both equipment and methodology.

\section{CONCLUSION}

It can be concluded, from the results obtained for the precision and accuracy, the FOM and the MDA, that the Cerenkov method, (LSC) direct method and LSC method with radiochemical separation are suitable for the for the determination of ${ }^{90} \mathrm{Sr}$ in water sample. The Cerenkov method and (LSC) direct method procedures require only reduction of the initial water solution, without radiochemical separation. When the sample presents other beta emitting radionuclides, the (LSC) method with radiochemical separation is required, without loss of quality of the results.

\section{REFERENCES}


1. L'ANnUnZIATA, M. F. Handbook of Radioactivity Analysis, $3^{\text {rd }}$ ed. Academic Press: Elsevier, 2012.

2. PACKARD. Handbook of Environmental Liquid Scintillation Spectrometry - A Compilation of Theory and Methods, ed. Packard - A Canberra Company, USA, 1994.

3. QUANTULUS. Instrument Manual-Wallac 1220 QuantulusTM Ultra Low Level Liquid Scintillation Spectrometer, ed. PerkinElmer, 2009.

4. HIDEX. Handbook 425-201 Automatic Liquid Scintillation Counter, Hidex 300SL, ed. Hidex, 2008.

5. PERKINELMER. Liquid Scintillation Analysis - Science and Technology, < $\underline{\text { http://www.perkinelmer.co.jp/Portals/0/resource/products_ls/ls_cocktails/pdf/LSABo }}$ oklet_012129_01.pdf, 2015.

6. CHYLIŃSKI, A.; BRODA, R.; RADOSZEWSKI, T. The National Standard Unit of Radionuclide Activity and the related standards in Poland, NUKLEONIKA, v. 48(1), p. 51-55, 2003.

7. CASSETTE, P.; GRIGORESCU, E. L.; RAZDOLESCU, A. C. Obtaining tritiated water standards using triple to double coincidence ratio (tdcr) method, Report WP7 IDRANAP 26-02, p. 2-7, 2002.

8. OLFERT, J, M.; DAI X.; KRAMER-TREMBLAY, S. Rapid determination of ${ }^{90} \mathrm{Sr} /{ }^{90} \mathrm{Y}$ in water samples by liquid scintillation and Cherenkov counting, Journal of Radioanalytical and Nuclear Chemistry, v.300, p.263-267, 2014.

9. CURRIE, L.A. Limits for qualitative detection and quantitative determination". Analytical Chemistry, v. 40, p.586-593, 1968.

10. VIANNA, M.E.; TAUHATA, L.; OLIVEIRA, J.P.; OLIVEIRA, A.E.; GARCIA, L. C.; CONCEIÇÃO, C.S.; CLAIN, A. F. Quality of radionuclide analysis in environmental samples. Applied Radiation and Isotopes, v.173/174, p.15-18, 1995.

11. TAUHATA, L.; VIANNA, M.E.C.M.; OLIVEIRA, A.E.; FERREIRA, A.C.M.; BRAGANÇA, M.J.C.S.; CLAIN, A. F. The influence of uncertainties of measurements in laboratory performance evaluation using an intercomparison program of 
radionuclide assays in environmental samples. Applied Radiation and Isotopes, v. 64, p.1174-1178, 2006. 